Article

\title{
Scrophularia koraiensis Nakai Attenuates Allergic Airway Inflammation via Suppression of NF- $K B$ and Enhancement of Nrf2/HO-1 Signaling
}

\author{
Tae-Yang Jung ${ }^{1}$, A Yeong Lee ${ }^{2}{ }^{(}$, Jun-Ho Song ${ }^{2}{ }^{(}$, Min Young Lee ${ }^{3}$, Je-Oh Lim ${ }^{1}$, Se-Jin Lee ${ }^{1}$, \\ Je-Won Ko ${ }^{1}$, Na-Rae Shin ${ }^{1}$, Jong-Choon Kim ${ }^{1}$, In-Sik Shin ${ }^{1, *}$ (i) and Joong-Sun Kim ${ }^{2, *}$ \\ 1 College of Veterinary Medicine (BK21 Plus Project Team), Chonnam National University, 77 Yongbong-ro, \\ Buk-gu, Gwangju 61186, Korea; jupiterriot@naver.com (T.-Y.J.); dvmljo@gmail.com (J.-O.L.); \\ xhdhksd1123@naver.com (S.-J.L.); rheoda@gmail.com (J.-W.K.); tlsskfo870220@gmail.com (N.-R.S.); \\ toxkim@jnu.ac.kr (J.-C.K.) \\ 2 Herbal Medicine Resources Research Center, Korea Institute of Oriental Medicine, 111 Geonjae-ro, Naju-si, \\ Jeollanam-do 58245, Korea; lay7709@kiom.re.kr (A.Y.L.); songjh@kiom.re.kr (J.-H.S.) \\ 3 College of Pharmacy, Research Institute of Pharmaceutical Sciences, Kyungpook National Univeristy, \\ 80 Daehak-ro, Buk-gu, Daegu 41566, Korea; vetmedic@knu.ac.kr \\ * Correspondence: dvmmk79@gmail.com (I.-S.S.); centraline@kiom.re.kr (J.-S.K.); \\ Tel.: +82-62-530-2835 (I.-S.S.); +82-61-338-7136 (J.-S.K.)
}

Received: 20 December 2019; Accepted: 22 January 2020; Published: 24 January 2020

check for updates

\begin{abstract}
Scrophularia koraiensis Nakai (Scrophulariaceae) is a medicinal herb that grows in Korea and which has been widely used to treat fever, edema, neuritis and laryngitis. Hence, we evaluated the anti-inflammatory and antioxidant effects of the ethanol extract (SKE) of S. koraiensis Nakai in an ovalbumin (OVA)-induced mouse model. We injected $20 \mu \mathrm{g}$ of OVA with $2 \mathrm{mg}$ of aluminum on day 0 and day 14 to induce allergic airway inflammation in six-week-old BALB/c mice, and mice were challenged with $1 \%$ OVA by nebulization for $1 \mathrm{~h}$ on days 21,22 , and 23 . SKE was orally administered at $20 \mathrm{mg} / \mathrm{kg}$ and $40 \mathrm{mg} / \mathrm{kg}$ from day 18 to 23, and its effects were compared with those of montelukast treatment. SKE significantly reduced proinflammatory cytokines, inflammatory cell counts, immunoglobulin-E, and airway hyperresponsiveness during the OVA-induced allergic airway inflammation model; it also reduced airway inflammation and mucus production. In addition, SKE reduced the OVA-induced nuclear factor kappa B (NF- $\mathrm{KB}$ ) phosphorylation in lung tissues while enhancing nuclear factor erythroid-derived 2-related factor (Nrf-2) and heme oxygenase-1 (HO-1) expression. In conclusion, SKE showed the protective effects on OVA-induced allergic airway inflammation via the suppression of NF-KB phosphorylation and the enhancement of the Nrf2/HO-1 signaling pathway. These results indicate that SKE is a potential therapeutic agent for allergic airway inflammation.
\end{abstract}

Keywords: Scrophularia koraiensis; Asthma; Nrf2; HO-1; NF-kB

\section{Introduction}

Asthma is a chronic inflammatory disease that affects 275 million people worldwide [1]. It is caused by exposure to specific allergens that aggravate inflammation, mucus hypersecretion, and airway hyperresponsiveness (AHR), resulting in the loss of normal lung function [2,3]. The clinical signs of asthma are wheezing, dyspnea, chest tightness, and cough due to limitations of airflow to the respiratory tracts [4]. The development of asthma is associated with the increased production of pro-inflammatory cytokines, chemokines, growth factors, and reactive oxygen species (ROS), which 
eventually lead to eosinophilia, AHR, and mucus overproduction [5-7]. There is an ongoing search for an effective asthma treatment that targets these varied pathways [8-10].

Oxidative stress is associated with the development of asthma [3]. Oxidative stress occurs because of an imbalance between external oxidation inducers and the antioxidant response within cells, resulting in the production of ROS [11], which induces asthmatic responses via the activation of inflammatory signaling $[12,13]$. The antioxidant signaling molecules, nuclear factor erythroid-derived 2-related factor 2 (Nrf2) and heme oxygenase-1 (HO-1), have been targeted by many researchers to counter excessive ROS during asthma development [14]. HO-1 is an antioxidant enzyme that is involved in oxidative stress, as well as in cell damage and inflammatory disease [15]. The expression of HO-1 inhibits the expression of nuclear factor kappa B (NF- $\mathrm{kB})$, thereby reducing the expression of inducible nitric oxide synthase (iNOS), resulting in an alteration in cytokine gene expression [16]. Therefore, HO-1 may exert anti-asthmatic effects by decreasing ROS production and inflammatory response.

The genus Scrophularia L., which belongs to the family Scrophulariaceae, consists of about 200 species. This genus is widely distributed across the temperate regions of the Northern Hemisphere. Moreover, various species of the genus Scrophularia have been used as therapeutic agents for fever, edema, constipation, neuritis and laryngitis $[17,18]$. One of these, Scrophularia koraiensis Nakai, is a rare medicinal herb that grows in Korea and has been used as an antipyretic and anti-inflammatory agent in the past [19]. The pharmacological properties of S. koraiensis have been described, but the effect of S. koraiensis on various diseases has not been studied. In the present study, we investigate the effects of S. koraiensis on ovalbumin-induced allergic airway inflammation, focusing on its anti-inflammatory and antioxidant properties.

\section{Materials and Methods}

\subsection{Animals}

Female-specific pathogen-free 6-week-old BALB/c mice were purchased from SAMTAKO (Osan, Korea). The mice were maintained under standard conditions (on a $12 \mathrm{~h}$ night/day cycle, at a humidity of $55 \pm 5 \%$ and temperature of $22 \pm 2{ }^{\circ} \mathrm{C}$ ) and fed ad libitum. All experiments were conducted according to a protocol approved by the Chonnam National University Institutional Animal Care and Use Committee. (CNU IACUC-YBR-2016-19, Gwangju, Korea)

\subsection{Materials and Instruments}

Scrophularia koraiensis Nakai was collected from the experimental field of the National Institute of Horticultural and Herbal Science (NIHHS, Chungcheongbuk-do, Korea, 36 56'24.7" N, 127 44'56.1" E), and medicinal parts and plant voucher specimens (2-18-0145, KIOM-2019-54) were deposited in the Korean Herbarium of Standard Herbal Resources (Index Herbariorum code: KIOM) at the Korea Institute of Oriental Medicine, Naju, Korea. S. koraiensis is distinguished from closely related species, Scrophularia buergeriana Miq. (Table S1) because it has an acuminate apex, regular double serrated margins, obtuse base, and acuminate calyx lobes (Figure S1). S. koraiensis (69.15 g) was refluxed in 70\% ethanol $(v / v)$ for $2 \mathrm{~h}$, and the was extract filtered and evaporated in vacuo. The yield of the $70 \%$ ethanol extract of $S$. koraiensis (SKE) was $43.96 \%(w / w)$ at $4{ }^{\circ} \mathrm{C}$.

Aucubin, harpagide, and harpagoside were purchased from Shanghai Sunny Biotech (Shanghai, China), and 8-acetyl harpagide and angoroside $\mathrm{C}$ were obtained from ChemFaces (Wuhan, Hubei, China). High performance liquid chromatography (HPLC) grade water, methanol, and acetonitrile were from Merck (Darmstadt, Germany). The HPLC system (Waters Corporation, Milford, MA, USA) was assembled by using the Waters e2695 Separation Module, a 2998 PDA detector, an Acquity QDa detector, and a micro-splitter (IDEX Health \& Science LLC, Oak Harbor. WA, USA). 


\subsection{HPLC analysis}

SKE $(110.5 \mathrm{mg})$ was dissolved in $10 \mathrm{~mL}$ of $70 \%$ ethanol and filtered through a $0.2 \mu \mathrm{m}$ syringe filter. The column was a Kinetex Biphenyl 100A $(4.6 \times 250$ mm, $5 \mu \mathrm{m}$, Phenomenex Inc., Torrance, CA, USA). The mobile phase was mixed with $0.05 \%$ aqueous formic acid (A), acetonitrile (B), and methanol (C), and a gradient program from $100 \% \mathrm{~A}(2 \mathrm{~min}) \rightarrow 96 \% \mathrm{~A}(3 \% \mathrm{~B}$ and $1 \% \mathrm{C}, 7 \mathrm{~min}) \rightarrow$ isocratic $85 \% \mathrm{~A}$ $(11 \% \mathrm{~B}$ and $4 \% \mathrm{C}$, from $15 \mathrm{~min}$ to $20 \mathrm{~min}) \rightarrow 70 \% \mathrm{~A}(23 \% \mathrm{~B}$ and $7 \% \mathrm{C}, 35 \mathrm{~min}) \rightarrow 50 \% \mathrm{~A}(35 \% \mathrm{~B}$ and $15 \% \mathrm{C}, 45 \mathrm{~min}) \rightarrow 30 \% \mathrm{~A}(50 \% \mathrm{~B}$ and $20 \% \mathrm{C}, 55 \mathrm{~min}$ ) was run. The flow rate was $0.8 \mathrm{~mL} / \mathrm{min}$, and the injected volume was $10 \mu \mathrm{L}$. The UV wavelength was monitored from 195 to $400 \mathrm{~nm}$. The QDa detector was set as follows: a nitrogen carrier gas, an electrospray interface (ESI) capillary of $0.8 \mathrm{kV}$, a $600^{\circ} \mathrm{C}$ probe temperature, a $15 \mathrm{~V}$ con voltage, a $120^{\circ} \mathrm{C}$ source temperature, and a 20:1 split. The mass range was from 300 to $850 \mathrm{~m} / \mathrm{z}$. The peaks were confirmed by using the standard compound peaks observed in the UV and MS spectra. The sample peaks were identified by comparing the retention time and molecular weight of each sample with that of the standard compound peaks.

\subsection{OVA-Induced Allergic Airway Inflammation Model}

To induce allergic airway inflammation in mice, intraperitoneal injections of OVA $(20 \mu \mathrm{g}$, Sigma-Aldrich, St. Louis, MO, USA) that was mixed with $2 \mathrm{mg}$ of aluminum hydroxide (Sigma-Aldrich) were administered on days 1 and 14 . Subsequently, mice received an OVA challenge ( $1 \% w / v$ in phosphate buffered saline (PBS)) for $1 \mathrm{~h}$ from days 21 to 23 . Montelukast (10 mg/ $/ \mathrm{kg}$ ) and SKE ( 20 and $40 \mathrm{mg} / \mathrm{kg}$ ) were administered by oral gavage from days 18 to 23. On day 24, AHR was evaluated by using whole-body plethysmography (OCP3000 instrument, Allmedicus, Seoul, Korea). AHR was evaluated following methylcholine inhalation $(0,10,20$ and $30 \mathrm{mg} / \mathrm{mL}$ in PBS) for $3 \mathrm{~min}$. The results of AHR were expressed as a dimensionless parameter, enhanced pause (Penh). The mice were divided into 5 groups $(n=5)$; NC (normal control; PBS sensitization, PBS challenge and PBS administration), OVA (OVA sensitization, OVA challenge and PBS administration), Mon (OVA sensitization, OVA challenge, and montelukast administration), SKE 20 and 40 (OVA sensitization, OVA challenge, and SKE administration (20 and $40 \mathrm{mg} / \mathrm{kg}$, respectively). The experimental procedure is shown in Figure 1.

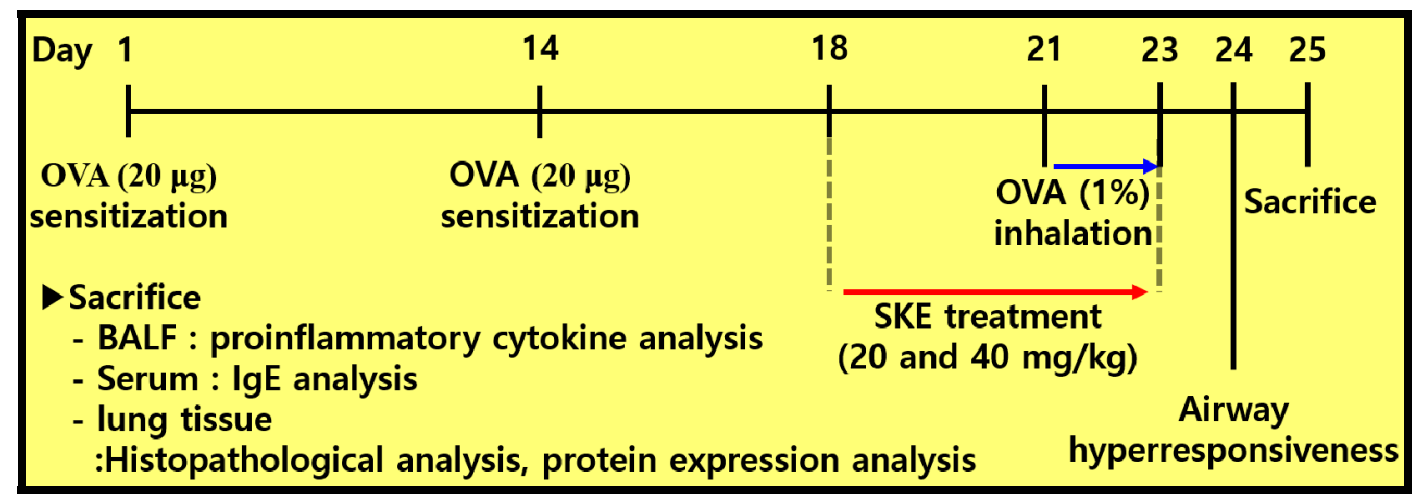

Figure 1. The experimental procedure.

\subsection{Measurement of Allergic Parameters in Bronchoalveolar Lavage Fluid (BALF) and Serum}

On day 25, mice were anesthetized with alfaxalone (Jurox, Rutherford, Australia), and blood samples were collected from the cauda vena cava. The blood samples were centrifuged for $20 \mathrm{~min}$ at $200 \mathrm{~g}$ to separate the serum. The total immunoglobulin E (IgE) and OVA-specific IgE were measured by an enzyme linked immunosorbent assay (ELISA) (BioLegend Inc., San Diego, CA, USA). To collect the bronchoalveolar lavage fluid (BALF), we performed tracheostomy in the mice and inserted endotracheal tubes. PBS $(0.7 \mathrm{~mL})$ was injected into the lung and removed via the tube, and the process was repeated once. The collected BALF was centrifuged $\left(200 \mathrm{~g}, 4{ }^{\circ} \mathrm{C}, 10 \mathrm{~min}\right)$. The supernatants were collected in new tubes for measuring pro-inflammatory cytokines interleukin (IL)- 5 and IL-13 by using ELISA. 
The remaining pellet was dissolved in $200 \mu \mathrm{L}$ of PBS, followed by centrifugation through Cytospin (Hanil Electric, Wonju, Korea) to attach inflammatory cells to the slides. Slides were stained by a Diff-Quik reagent (Sysmex, Kobe, Japan) for counting the different cells in the BALF.

\subsection{Histopathology of Lung Tissue}

To perform histological examinations, the left lung was fixed in formalin after BALF sampling. The lung tissue samples were embedded in paraffin blocks, and $4 \mu \mathrm{m}$ sections were prepared. Tissues were stained in hematoxylin and eosin (H and E, Sigma-Aldrich) and periodic acid-Schiff (PAS, IMEB Inc., San Marcos, CA, USA) to evaluate airway inflammatory responses and mucus production, respectively. A quantitative analysis of inflammation and mucus production in lung tissue was performed with the use of an image analyzer (IMT i-Solution software, IMT i-Solution Inc., Vancouver, BC, Canada).

Immunohistochemistry (ICH) was performed to evaluate inducible nitric oxide synthase (iNOS) and HO-1 expression in lung tissue by using a commercial kit (Vector Laboratories, Burlingame, CA, USA). The anti-mouse iNOS antibody (diluted 1:200, Abcam, Cambridge, UK) and anti-mouse HO-1 (diluted 1:200, Abcam) were used as primary antibodies. The slides were examined under a light microscope (Leica, Wetzlar, Germany) in a completely blinded manner.

\subsection{Western Blotting}

To identify the proteins and signaling pathways within the lung tissue, the right lung was homogenized with a tissue lysis/extraction reagent (Sigma-Aldrich). The total protein content of each sample was determined by using the Bradford assay (Bio-Rad Laboratories, Hercules, CA, USA). A total of $30 \mu \mathrm{g}$ protein was electrophoresed by using $10 \%$ SDS-polyacrylamide gel and then transferred to a polyvinyl difluoride membrane. The membrane was blocked with $5 \%$ skim milk for $1 \mathrm{~h}$ and incubated overnight at $4{ }^{\circ} \mathrm{C}$ with the following primary antibodies: p-65 (diluted 1:1000, Abcam), phosphorylated-p65 (diluted 1:1000, Abcam), iNOS (diluted 1:1000, Abcam), HO-1 (diluted 1:1000, Cell Signaling, Beverly, MA, USA), Nrf2 (diluted 1:1000, Cell Signaling), $\beta$-actin (diluted 1:1000, Cell Signaling), lamine B1 (diluted 1:1000, Abcam). After washing three times with tris-buffered saline (TBS) that was supplemented with Tween 20, the horseradish peroxidase (HRP)-conjugated secondary antibody was diluted to 1:3000 and incubated for $1 \mathrm{~h}$. Membranes were washed again, and binding was detected by using an enhanced chemiluminescence kit (Thermo-Scientific, Waltham, MA, USA). The density of each protein band was evaluated by using ChemiDoc (Bio-Rad Laboratories, Hercules, CA, USA).

\subsection{Statistical Analysis}

All data are expressed as mean \pm standard deviation. Statistical evaluation was performed by using analysis of variance (ANOVA) followed by Dunnett's post-hoc adjustments. A value of $p<0.05$ was determined to be significant.

\section{Results}

\subsection{Identification of Compounds within SKE by HPLC Analysis}

The HPLC chromatograms of SKE at 200 and $280 \mathrm{~nm}$ are shown in Figure 2. Aucubin $(15.70 \pm 0.259 \mu \mathrm{g} / \mathrm{mg})$, harpagide $(12.22 \pm 0.131 \mu \mathrm{g} / \mathrm{mg}), 8$-acetyl harpagide $(1.64 \pm 0.015 \mu \mathrm{g} / \mathrm{mg})$, angoroside $C(2.90 \pm 0.014 \mu \mathrm{g} / \mathrm{mg})$, and harpagoside $(6.75 \pm 0.049 \mu \mathrm{g} / \mathrm{mg})$ were detected at approximately $11.5,13.4,19.9,37.2$, and $42.2 \mathrm{~min}$, respectively. With the exception of harpagoside, which was detected at $280 \mathrm{~nm}$, the four other compounds were detected at $200 \mathrm{~nm}$ (Figure 2a,b). The predominant compounds aucubin and harpagide were classified a iridoids. The five peaks of SKE were also analyzed for their UV wavelength and molecular weight. Their UV spectra were $\lambda_{\max }=195.3,195.7,195.7,198.0$, and $279.5 \mathrm{~nm}$. In positive mode, the five compounds were confirmed as follows: aucubin $\left[\mathrm{M}+\mathrm{Na}^{+}\right]^{+}=369.1 \mathrm{~m} / z$, harpagide $\left[\mathrm{M}+\mathrm{Na}^{+}+\mathrm{OH}^{-}\right]^{+}=387.1 \mathrm{~m} / \mathrm{z}$, 8-acetyl harpagide $\left[\mathrm{M}+\mathrm{Na}^{+}\right]^{+}=429.1 \mathrm{~m} / \mathrm{z}$, angoroside $\mathrm{C}$ 
$\left[\mathrm{M}+\mathrm{Na}^{+}\right]^{+}=807.3 \mathrm{~m} / \mathrm{z}$, and harpagoside $\left[\mathrm{M}+\mathrm{Na}^{+}\right]^{+}=517.2 \mathrm{~m} / \mathrm{z}$ (Figure $2 \mathrm{c}$ ). The total ion chromatogram (TIC) and extracted ion chromatogram (XIC) were compared to verify the five compounds that were detected in SKE on the chromatogram. In the mass range of $300-850 \mathrm{~m} / \mathrm{z}$, the TIC was a black line and its XIC values were expressed as follows: Red was aucubin as $369.1 \mathrm{~m} / \mathrm{z}$, blue was harpagide as $387.1 \mathrm{~m} / \mathrm{z}$, green was 8-acetyl harpagide as $429.3 \mathrm{~m} / \mathrm{z}$, cyan was angoroside $\mathrm{C}$ as $807.3 \mathrm{~m} / \mathrm{z}$, and pink was harpagoside as $517.2 \mathrm{~m} / \mathrm{z}$ (Figure 2d).
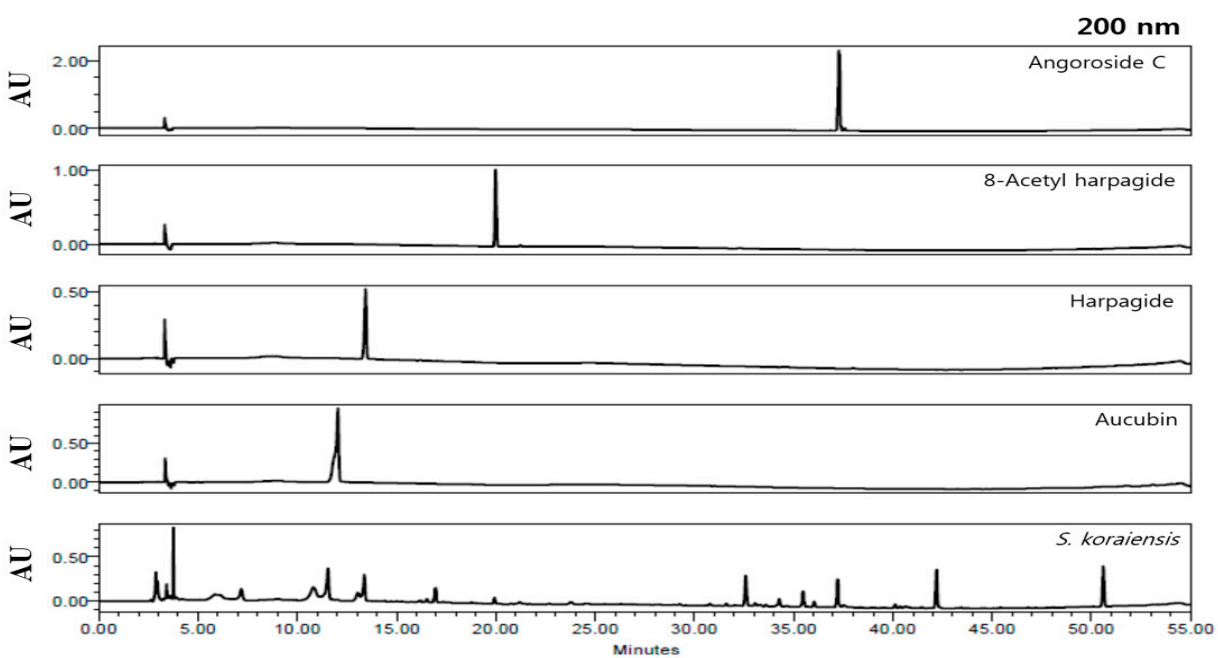

(a)
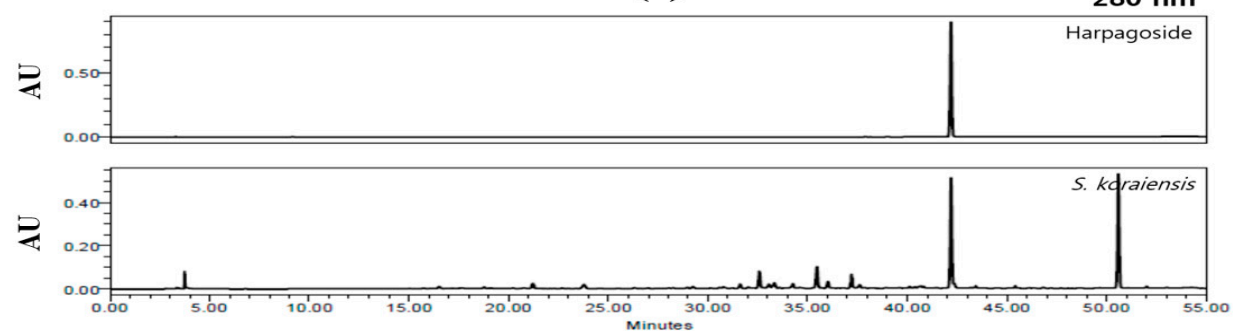

(b)

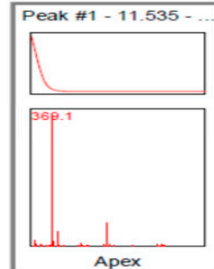

Apex

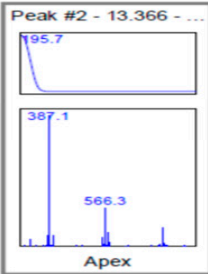

Apex

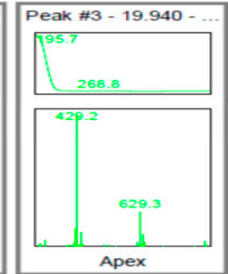

(c)
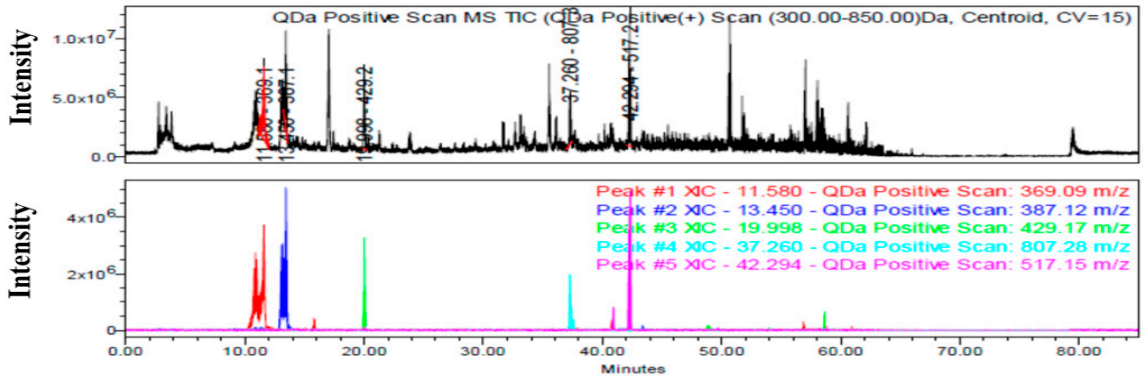

(d)

Figure 2. Chromatogram of S. koraiensis at $200 \mathrm{~nm}$ (a) and $280 \mathrm{~nm}$ (b), UV spectra and MS spectra of peaks according to retention time (c), total ion chromatogram (TIC) and extracted ion chromatogram $(\mathrm{XIC})(\mathbf{d})$. 


\subsection{Effect of SKE on AHR and Inflammatory Cell Count During OVA-Induced Allergic Airway Inflammation}

The OVA-challenged group showed a marked elevation of AHR in comparison to the PBS-challenged controls (Figure 3a). Montelukast treatment significantly reduced AHR compared to the OVA-challenged group that received PBS treatment. The effect of SKE treatment was similar to that of the montelukast treatment, inducing a marked decline of AHR in comparison to that in the OVA-challenged group that received PBS treatment. This reduction was more marked after the high dose $(40 \mathrm{mg} / \mathrm{kg})$ SKE treatment.

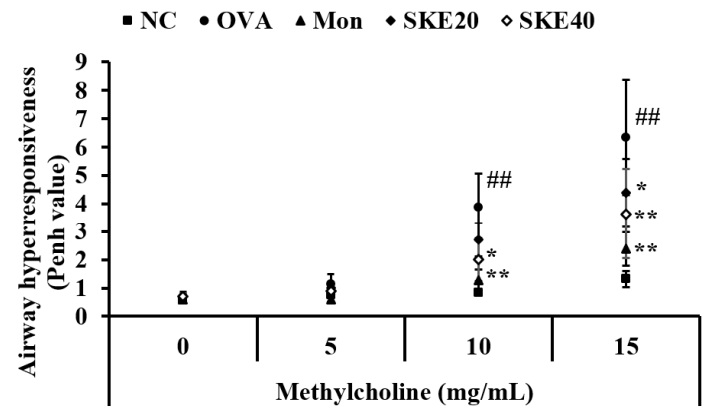

(a)

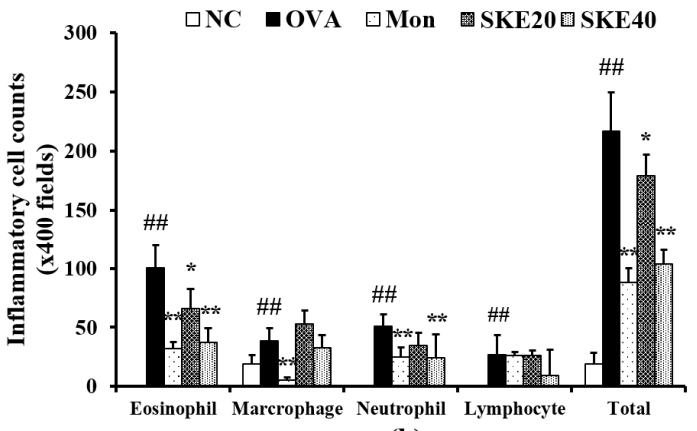

(b)

Figure 3. SKE (ethanol extract of S. koraiensis) reduced airway hyperresponsiveness (AHR) and inflammatory cell counts during ovalbumin (OVA)-induced allergic airway inflammation. The bronchoalveolar lavage fluid (BALF) was stained with a Diff-Quik agent for cell counting, and AHR was measured by using whole-body plethysmography. (a) AHR and (b) inflammatory cell counts in the BALF. NC (normal control), PBS (phosphate buffered saline) treatment and PBS sensitization/challenge; OVA, PBS treatment and OVA sensitization/challenge; Mon: montelukast treatment and OVA sensitization/challenge; SKE 20 and 40, SKE treatment (20 and $40 \mathrm{mg} / \mathrm{kg}$, respectively) and OVA sensitization/challenge. Values are shown as the mean $\pm \operatorname{SD}(n=5)$. \#\# $p<0.01$ versus NC; $* * * *<0.05$ and 0.01 versus OVA.

The OVA challenge led to marked increases in inflammatory cell in the BALF—of eosinophils, in particular (Figure 3b). Both the montelukast treatment and SKE treatments reduced the OVA-induced inflammatory cell increases. The high dose $(40 \mathrm{mg} / \mathrm{kg})$ SKE treatment induced the most significant decline in inflammatory cell counts.

\subsection{Effect of SKE on Pro-Inflammatory Cytokines and IgE Levels During OVA-Induced Allergic Airway Inflammation}

The OVA challenge induced marked elevations of IL-5 and IL-13 in the BALF compared to the PBS-challenged controls (Figure 4a,b, respectively). Both the montelukast and SKE treatments led to a significant reduction in pro-inflammatory cytokines as well as decline in the total $\operatorname{IgE}$ and OVA-specific IgE (Figure 4c,d, respectively).

\subsection{Effect of SKE on Airway Inflammation and Mucus Production During OVA-Induced Allergic Airway Inflammation}

The OVA challenge markedly increased inflammatory cell accumulation in lung tissues compared to the PBS-challenged controls (Figure 5a). Both the montelukast and SKE treatments significantly reduced the OVA-induced inflammatory cell accumulation. Similarly, both treatments also alleviated the increases in the mucus production that was induced by OVA (Figure $5 b$ ). 

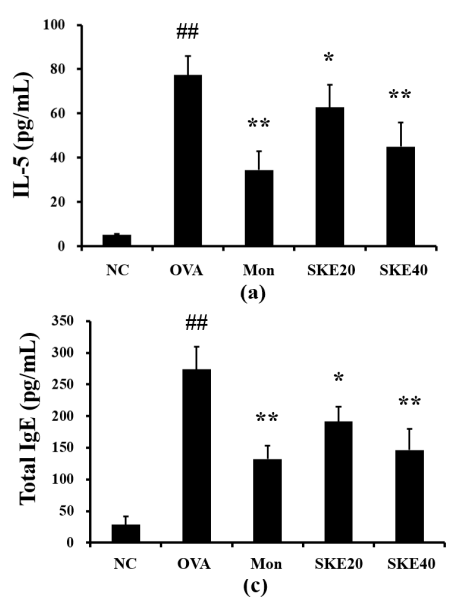
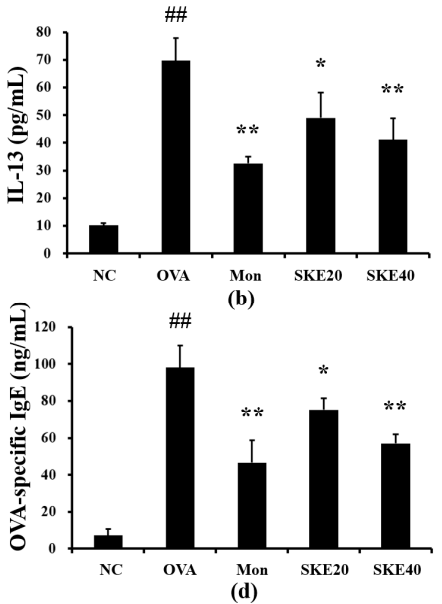

Figure 4. SKE decreased proinflammatory cytokines and immunoglobulin E (IgE) during OVA-induced allergic airway inflammation. Interleukin (IL)-5, IL-13, total IgE, and OVA-specific IgE were determined with commercial ELISA kits. (a) IL-5, (b) IL-13, (c) total IgE, (d) OVA-specific IgE. NC, PBS treatment and PBS sensitization/challenge; OVA, PBS treatment and OVA sensitization/challenge; Mon: montelukast treatment and OVA sensitization/challenge; SKE 20 and 40, SKE treatment (20 and $40 \mathrm{mg} / \mathrm{kg}$, respectively) and OVA sensitization/challenge. Values are shown as the mean $\pm \operatorname{SD}(n=5)$. \#\# $p<0.01$ versus NC; $* * * *<0.05$ and 0.01 versus OVA.

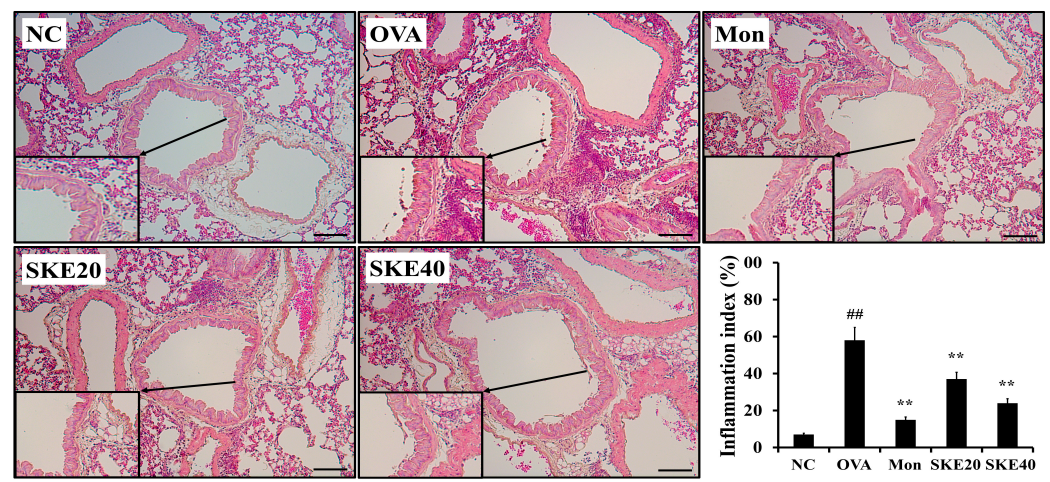

(a)

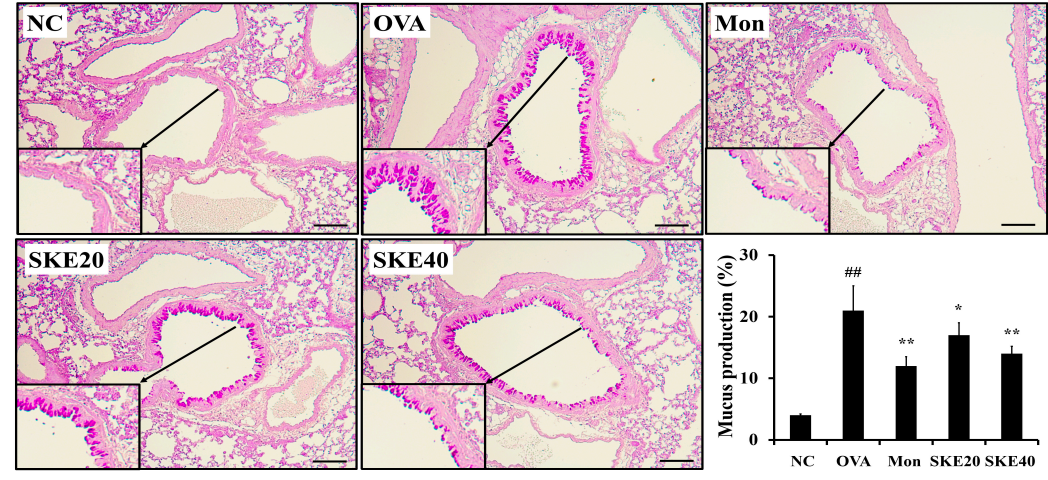

(b)

Figure 5. SKE attenuated airway inflammation and mucus production during OVA-induced allergic airway inflammation. Lung tissue samples were stained with hematoxylin and eosin ( $\mathrm{H}$ and $\mathrm{E})$ and Diff-Quik agents. (a) Airway inflammation and (b) mucus production. The quantitative analysis of airway inflammation and mucus production were measured by an image analyzer. NC, PBS treatment and PBS sensitization/challenge; OVA, PBS treatment and OVA sensitization/challenge; Mon: montelukast treatment and OVA sensitization/challenge; SKE 20 and 40, SKE treatment (20 and $40 \mathrm{mg} / \mathrm{kg}$, respectively) and OVA sensitization/challenge. Scale bars indicate $50 \mu \mathrm{m}$. Values are shown as the mean $\pm \mathrm{SD}(n=5)$. \#\# $p<0.01$ versus $\mathrm{NC} ;{ }^{*}{ }^{* *} p<0.05$ and 0.01 versus OVA. 


\subsection{Effect of SKE on NF-אB Phosphorylation and iNOS Expression During OVA-Induced Allergic Airway Inflammation}

The OVA challenge led to an increased NF- $\mathrm{kB}$ phosphorylation compared to the PBS-challenged controls (Figure 6). Montelukast and SKE treatments reduced the increases in NF- $\kappa B$ phosphorylation. iNOS expression in the lung tissue was also markedly increased in response to the OVA challenge, and this was reduced by SKE treatment, as well as the results of IHC (Figure 7).

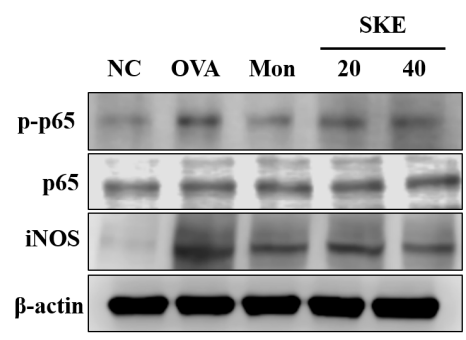

(a)

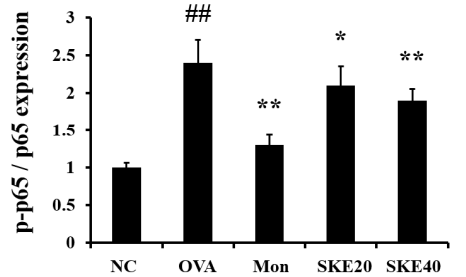

(b)

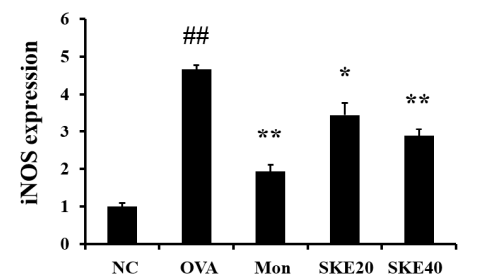

(c)

Figure 6. SKE decreased nuclear factor kappa B (NF-kB) phosphorylation and inducible nitric oxide synthase (iNOS) expression during OVA-induced allergic airway inflammation. NF- $\mathrm{B}$ phosphorylation and iNOS expression were measured by western blotting. (a) Protein expression on gel, (b) relative p-p65/p65 expression value, and (c) relative iNOS expression value. NC, PBS treatment and PBS sensitization/challenge; OVA, PBS treatment and OVA sensitization/challenge; Mon: montelukast treatment and OVA sensitization/challenge; SKE 20 and 40, SKE treatment (20 and $40 \mathrm{mg} / \mathrm{kg}$, respectively) and OVA sensitization/challenge. Values are shown as the mean $\pm \operatorname{SD}(n=5)$. \#\# $p<0.01$ versus NC; $* * * *<0.05$ and 0.01 versus OVA.

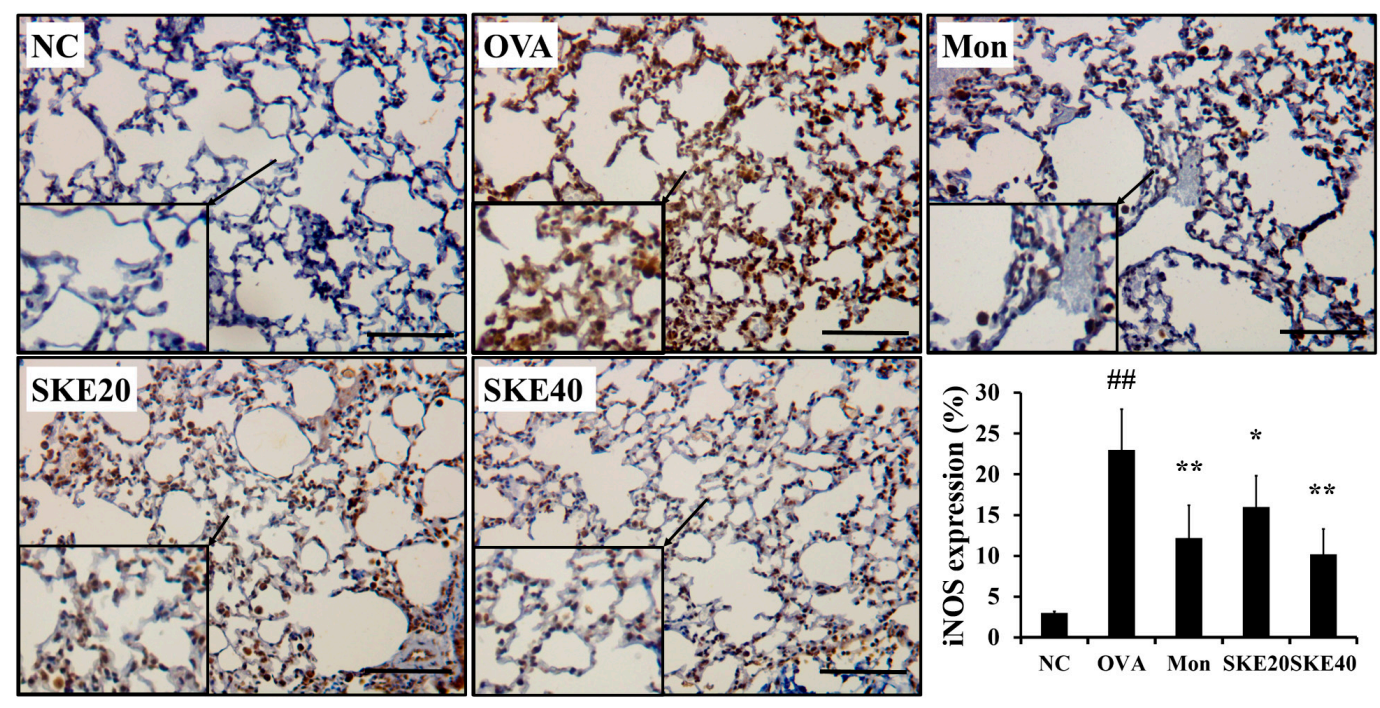

Figure 7. SKE reduced iNOS expression in the lungs during OVA-induced allergic airway inflammation. The expression of iNOS on lung tissue was determined by immunohistochemistry (IHC). NC, PBS treatment and PBS sensitization/challenge; OVA, PBS treatment and OVA sensitization/challenge; Mon: montelukast treatment and OVA sensitization/challenge; SKE 20 and 40, SKE treatment (20 and $40 \mathrm{mg} / \mathrm{kg}$, respectively) and OVA sensitization/challenge. Scale bars indicate $50 \mu \mathrm{m}$. Values are shown as the mean $\pm \mathrm{SD}(n=5)$. \#\# $p<0.01$ versus $\mathrm{NC}^{*}{ }^{* * *} p<0.05$ and 0.01 versus OVA.

\subsection{Effect of SKE on Nrf2/HO-1 Signaling During OVA-Induced Allergic Airway Inflammation}

The OVA challenge increased nuclear Nrf2 expression, whereas the PBS challenge did not (Figure 8a,b). The montelukast and SKE treatments elevated the nuclear Nrf2 expression in comparison to the OVA challenge. Similarly, HO-1 expression was elevated in response to the OVA challenge and 
more elevated by the SKE treatment (Figure 8a,c). These results were consistent with the results of IHC. The OVA challenge markedly increased the HO-1 expression in lung tissues compared to the PBS-challenged controls, and these increases were greater with the SKE treatment (Figure 9).

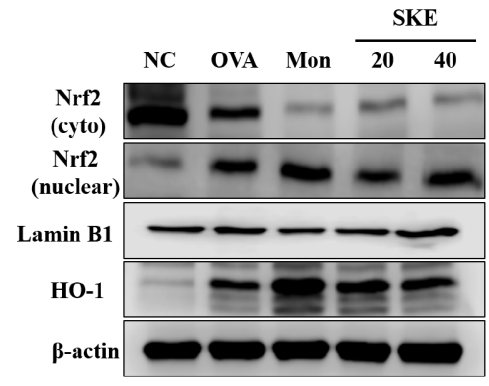

(a)

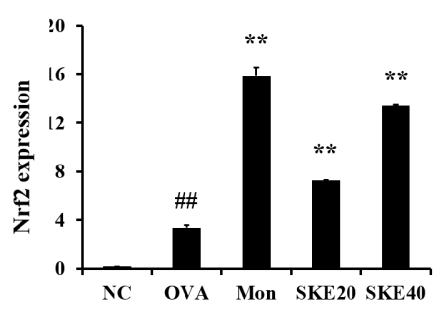

(b)

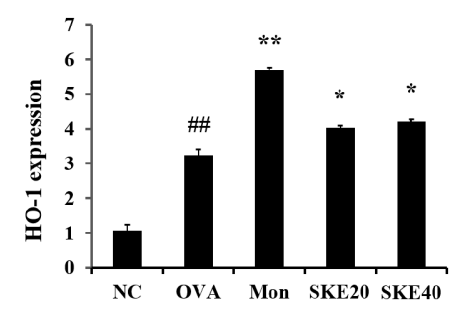

(c)

Figure 8. SKE enhanced Nrf2/HO-1 (nuclear factor erythroid-derived 2-related factor/heme oxygenase-1) signaling during the OVA-induced allergic airway inflammation model. The expression of Nrf2 and HO-1 were measured by western blotting. (a) Protein expression on gel, (b) relative nucleus/cytoplasm of Nrf2 expression value, (c) relative HO-1 expression value. NC, PBS treatment and PBS sensitization/challenge; OVA, PBS treatment and OVA sensitization/challenge; Mon: montelukast treatment and OVA sensitization/challenge; SKE 20 and 40, SKE treatment (20 and $40 \mathrm{mg} / \mathrm{kg}$, respectively) and OVA sensitization/challenge. Values are shown as the mean $\pm \operatorname{SD}(n=5)$. \#\# $p<0.01$ versus NC; **** $p<0.05$ and 0.01 versus OVA.
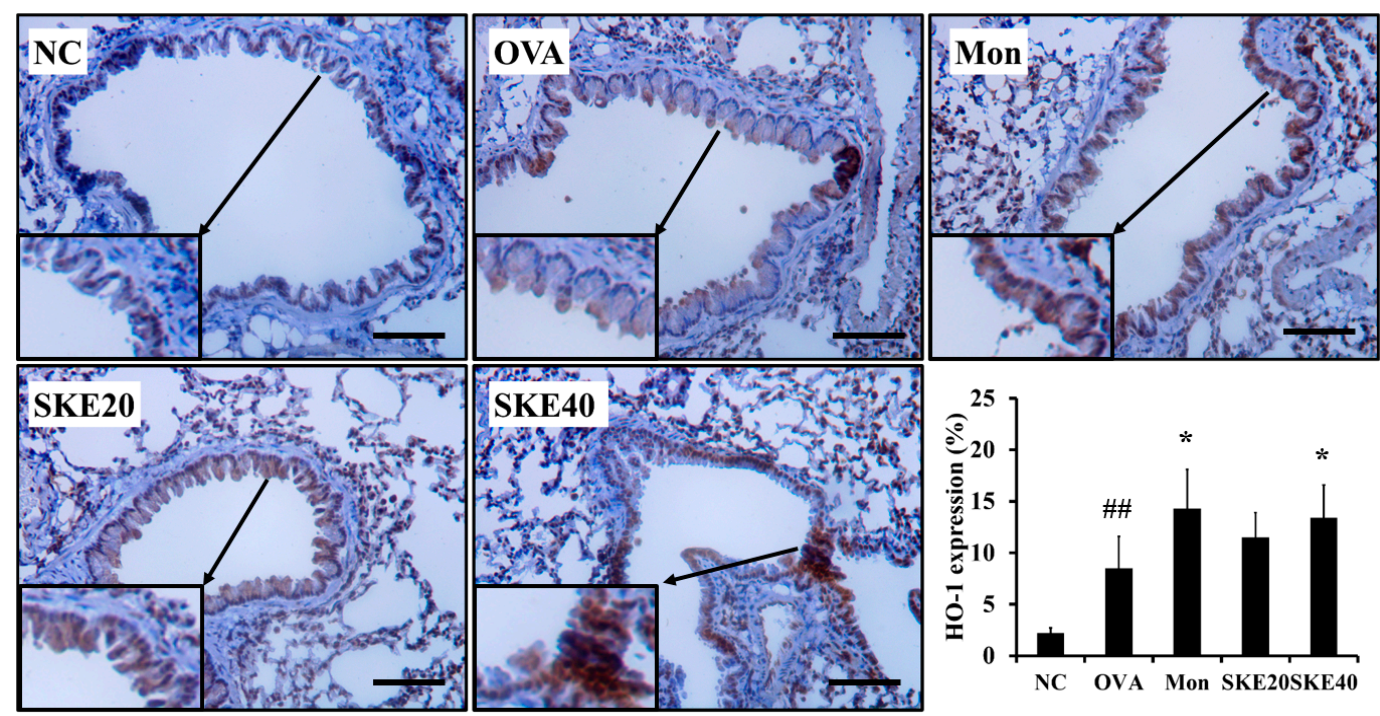

Figure 9. SKE reduced HO-1 expression in the lungs during OVA-induced allergic airway inflammation. The expression of HO-1 on lung tissue was determined by IHC. NC, PBS treatment and PBS sensitization/challenge; OVA, PBS treatment and OVA sensitization/challenge; Mon: montelukast treatment and OVA sensitization/challenge; SKE 20 and 40, SKE treatment (20 and $40 \mathrm{mg} / \mathrm{kg}$, respectively) and OVA sensitization/challenge. Scale bars indicate $50 \mu \mathrm{m}$. Values are shown as the mean $\pm \operatorname{SD}(n=5)$. \#\# $p<0.01$ versus $\mathrm{NC} ;{ }^{*} p<0.05$ versus OVA.

\section{Discussion}

Asthma is characterized by the chronic inflammation of the respiratory system, mucus production, and AHR, all of which result in airway obstruction and the interruption of normal airflow [20,21]. In this study, we investigated the protective effects of SKE in an OVA-induced allergic airway inflammation model, focusing on its anti-inflammatory and antioxidant properties. The SKE treatment decreased inflammatory cell counts and reduced IL-5, IL-13, total IgE, and OVA-specific IgE production, 
together with a reduction in AHR. In addition, SKE reduced NF-KB phosphorylation and iNOS expression in lungs while enhancing Nrf2/HO-1 signaling.

Elevated eosinophil numbers are considered major biomarkers of asthma [22]. Eosinophilia during asthma development is associated with pro-inflammatory cytokines such as IL-4, IL-5 and IL-13 [23,24]. These cytokines are involved in driving the maturation, activation, and accumulation of eosinophils within damaged lung lesions, leading to IgE production, airway inflammation, mucus overproduction, and AHR $[25,26]$. Therefore, the modulation of pro-inflammatory cytokines is regarded as an important strategy for controlling asthma. In this study, SKE significantly decreased the number of eosinophils during OVA-induced allergic airway inflammation and was accompanied by a reduction in pro-inflammatory cytokines, IgE and AHR. These responses to the SKE treatment were consistent with histopathological examinations that indicated a reduction in airway inflammation and mucus production. These results indicate that SKE has an anti-asthmatic effect.

The phosphorylation of NF- $\mathrm{KB}$ plays a major role in allergic responses during allergic asthma. During the development of asthma, phosphorylated NF- $\mathrm{KB}$ that is induced by various stimuli translocates to the nucleus and upregulates the expression of various pro-inflammatory genes [27]. In particular, the upregulation of iNOS expression downstream of NF- $\mathrm{kB}$ phosphorylation promotes inflammation via the increased production of pro-inflammatory mediators including cytokines, chemokines, and molecules that cause oxidative stress $[28,29]$. iNOS expression has been shown to be increased and to be accompanied by the upregulation of NF- $\mathrm{KB}$ in patients with asthma [30]. During the development of asthma, iNOS expression leads to nitric oxide (NO) production, further exacerbating asthma, as NO is an oxidative stress factor [31,32]. Therefore, the modulation of NF- $\mathrm{kB}$ and iNOS signaling is regarded as an important strategy for treating asthma. In this study, SKE significantly reduced iNOS expression and reduced NF-KB phosphorylation during OVA-induced allergic airway inflammation. These results indicate that the anti-asthmatic effects of SKE could be due to modulation of NF-KB phosphorylation and iNOS expression.

Oxidative stress is associated with the development of asthma, and various antioxidative responses are produced to limit oxidative stress-related damage. HO- 1 is one of the enzymes that is induced by oxidative stress to act as host defense to reduce oxidative stress and associated inflammatory responses [33]. HO-1 expression is modulated by various transcription factors including Nrf2. Oxidative stress causes Nrf2 to dissociate from Kelch-like epichlorohydrin-associated proteins (Keap 1) and translocate into the nucleus, where it directly binds the antioxidant response elements (AREs) [34]. These events lead to the eventual increase in HO-1 expression, boosting the antioxidant defense system [35]. Many studies have also demonstrated that Nrf2/HO-1 signaling is involved in inflammatory responses. The upregulation of Nrf2/HO-1 signaling suppresses inflammatory responses via the suppression of NF- $\mathrm{KB}$ signaling in a number of inflammatory diseases $[33,36]$. In this study, SKE induced the nuclear translocation of Nrf2 during OVA-induced allergic airway inflammation, which resulted in the overexpression of HO-1. These results indicate that SKE could attenuate asthmatic response via the activation of Nrf2/HO-1 signaling. The antioxidant effect of SKE has also been supported by previous studies $[37,38]$. In other members of the Scrophularia genus, Scrophularia umbrosa has shown strong free radical scavenging activity [37], and S. buergeriana has been shown to enhance the Nrf2/HO-1 signaling pathway [38].

In this study, SKE showed an anti-asthmatic effect on an OVA-induced asthma model. However, the effects of SKE are somewhat smaller than the effects of montelukast. Montelukast, a leukotriene receptor antagonist, is clinically used to relieve allergic airway inflammation [39]. The difference between SKE and montelukast is considered to be due to the chemical composition of each material. In this study, montelukast was used as a single compound, but SKE was used as an extract that consisted of various compounds. Therefore, further studies are needed to find the active single compound of SKE and investigate its efficacy for asthma. 


\section{Conclusions}

In conclusion, SKE reduced eosinophilia and pro-inflammatory cytokines production, decreased IgE levels, and alleviated AHR during OVA-induced allergic airway inflammation, as supported by histological evidence of reductions in airway inflammation and mucus secretion. These anti-asthmatic effects of SKE were closely associated with the suppression of NF- $\mathrm{kB}$ phosphorylation and the activation of Nrf2/HO-1 signaling. Our results suggest that SKE may be an effective therapeutic for suppressing asthma development.

Supplementary Materials: The following are available online at http://www.mdpi.com/2076-3921/9/2/99/s1, Figure S1: Stereomicroscope micrographs showing the external morphology of Scrophularia koraiensis Nakai., Table S1: Comparison of morphological characteristics in two species in Scrophularia.

Author Contributions: Conceptualization, I.-S.S. and J.-S.K.; methodology, T.-Y.J. and A.Y.L.; formal analysis, T.-Y.J., A.Y.L., M.Y.L., J.-O.L., S.-J.L., J.-W.K., and N.-R.S.; investigation, T.-Y.J. and A.Y.L.; resources, J.-H.S.; data curation, I.-S.S. and J.-S.K.; writing—original draft preparation, T.-Y.J.; writing—review and editing, J.-C.K., I.-S.S. and J.-S.K.; supervision, J.-S.K.; project administration, T.-Y.J. and S.-J.L.; funding acquisition, J.-S.K. All authors have read and agreed to the published version of the manuscript.

Funding: This research was supported by projects entitled "Evaluation of Effectiveness of Alternative Herbal Medicine Resources" (grant number: K18402) and "Applicational Development of Standardized Herbal Resources" (grand number: KSN 1911420) from the Korea Institute of Oriental Medicine (KIOM).

Acknowledgments: We thank the Korean Herbarium of Standard Herbal Resources (herbarium code KIOM) for the provision of materials.

Conflicts of Interest: The authors declare no conflict of interest.

\section{References}

1. Huang, C.C.; Chang, P.H.; Wu, P.W.; Wang, C.H.; Fu, C.H.; Huang, C.C.; Tseng, H.J.; Lee, T.J. Impact of nasal symptoms on the evaluation of asthma control. Medicine 2017, 96, e6147. [CrossRef] [PubMed]

2. Shin, N.R.; Ryu, H.W.; Ko, J.W.; Park, S.H.; Yuk, H.J.; Kim, H.J.; Kim, J.C.; Jeong, S.H.; Shin, I.S. Artemisia argyi attenuates airway inflammation in ovalbumin-induced asthmatic animals. J. Ethnopharmacol. 2017, 209, 108-115. [CrossRef] [PubMed]

3. Sahiner, U.M.; Birben, E.; Erzurum, S.; Sackesen, C.; Kalayci, Ö. Oxidative stress in asthma: Part of the puzzle. Pediatr. Allergy Immunol. 2018, 29, 789-800. [CrossRef] [PubMed]

4. Sanchez-Solis, M. Early lung function and future asthma. Front. Pediatr. 2019, 7, 253. [CrossRef]

5. Gauthier, M.; Ray, A.; Wenzel, S.E. Evolving concepts of asthma. Am. J. Respir. Crit. Care Med. 2015, 192, 660-668. [CrossRef]

6. Akiki, Z.; Rava, M.; Diaz Gil, O.; Pin, I.; le Moual, N.; Siroux, V.; Guerra, S.; Chamat, S.; Matran, R.; Fitó, M.; et al. Serum cytokine profiles as predictors of asthma control in adults from the EGEA study. Respir. Med. 2017, 125, 57-64. [CrossRef]

7. Fahy, J.V. Type 2 inflammation in asthma-Present in most, absent in many. Nat. Rev. Immunol. 2015, 15, 57-65. [CrossRef]

8. Shergis, J.L.; Wu, L.; Zhang, A.L.; Guo, X.; Lu, C.; Xue, C.C. Herbal medicine for adults with asthma: A systematic review. J. Asthma 2016, 53, 650-659. [CrossRef]

9. Hu, L.; Li, L.; Zhang, H.; Li, Q.; Jiang, S.; Qiu, J.; Sun, J.; Dong, J. Inhibition of airway remodeling and inflammatory response by Icariin in asthma. BMC Complement. Altern. Med. 2019, 19, 316. [CrossRef]

10. Lee, J.W.; Min, J.H.; Kim, M.G.; Kim, S.M.; Kwon, O.K.; Oh, T.K.; Lee, J.K.; Kim, T.Y.; Lee, S.W.; Choi, S.; et al. Pistacia weinmannifolia root exerts a protective role in ovalbumin-induced lung inflammation in a mouse allergic asthma model. Int. J. Mol. Med. 2019, 44, 2171-2180. [CrossRef]

11. Thannickal, V.J.; Fanburg, B.L. Reactive oxygen species in cell signaling. Am. J. Physiol. Lung Cell. Mol. Physiol. 2000, 279, L1005-L1028. [CrossRef] [PubMed]

12. Bullone, M.; Lavoie, J.P. The Contribution of Oxidative Stress and Inflamm-Aging in Human and Equine Asthma. Int. J. Mol. Sci. 2017, 18, 2612. [CrossRef] [PubMed]

13. Belenky, P.; Collins, J.J. Antioxidant strategies to tolerate antibiotics. Science 2011, 334, 915-916. [CrossRef] 
14. Rahman, I.; Biswas, S.K.; Kode, A. Oxidant and antioxidant balance in the airways and airway diseases. Eur. J. Pharmacol. 2006, 533, 222-239. [CrossRef] [PubMed]

15. Ryter, S.W.; Otterbein, L.E.; Morse, D.; Choi, A.M. Heme oxygenase/carbon monoxide signaling pathways: Regulation and functional significance. Mol. Cell. Biochem. 2002, 234-235, 249-263. [CrossRef]

16. Zhang, Z.H.; Zhu, W.; Ren, H.Z.; Zhao, X.; Wang, S.; Ma, H.C.; Shi, X.L. Mesenchymal stem cells increase expression of heme oxygenase-1 leading to anti-inflammatory activity in treatment of acute liver failure. Stem Cell Res. Ther. 2017, 8, 70. [CrossRef]

17. Xiao, S.; Tolga, E.; Henry, J.G.; Thomas, E. Effects of Scrophularia ningpoensis Hemsl. on inhibition of proliferation, apoptosis induction and NF-KB signaling of immortalized and cancer cell lines. Pharmaceutical 2012, 5, 189-208. [CrossRef]

18. Mitsuo, M.; Yoshiharu, O.; Sei-ichi, N.; Hiromu, K. Suppression of SOS-inducing activity of chemical mutagens by cinnamic acid derivatives from Scrophulia ningpoensis in the Salmonella typhimurium TA1535/pSK1002 umu test. J. Agric. Food Chem. 1998, 46, 904-910. [CrossRef]

19. Han, K.S.; So, S.K.; Lee, C.H.; Kim, M.Y. Taxonomy of the genus Scrophularia (Scrophulariaceae) in Korea. Korean J. Plant Taxon. 2009, 39, 237-246. [CrossRef]

20. Ko, J.W.; Shin, N.R.; Park, S.H.; Cho, Y.K.; Kim, J.C.; Seo, C.S.; Shin, I.S. Genipin inhibits allergic responses in ovalbumin-induced asthmatic mice. Int. Immunopharmacol. 2017, 53, 49-55. [CrossRef]

21. Tran, T.N.; Zeiger, R.S.; Peters, S.P.; Colice, G.; Newbold, P.; Goldman, M.; Chipps, B.E. Overlap of atopic, eosinophilic, and Th2-high asthma phenotypes in a general population with current asthma. Ann. Allergy Asthma Immunol. 2016, 116, 37-42. [CrossRef]

22. Shrestha Palikhe, N.; Bosonea, A.M.; Laratta, C.; Gandhi, V.D.; Nahirney, D.; Hillaby, A.; Bowen, M.; Bhutani, M.; Mayers, I.; Cameron, L.; et al. Stability of peripheral blood immune markers in patients with asthma. Allergy Asthma Clin. Immunol. 2019, 15, 30. [CrossRef]

23. Narendra, D.K.; Hanania, N.A. Targeting IL-5 in COPD. Int. J. Chronic Obstr. Pulm. Dis. 2019, 14, $1045-1051$. [CrossRef] [PubMed]

24. Robinson, D.S.; Hamid, Q.; Ying, S.; Tsicopoulos, A.; Barkans, J.; Bentley, A.M.; Corrigan, C.; Durham, S.R.; Kay, A.B. Predominant Th2-like bronchoalveolar T-lymphocyte population in atopic asthma. N. Engl. J. Med. 1992, 326, 298-304. [CrossRef] [PubMed]

25. Montero-Pérez, O.; Contreras-Rey, M.B.; Sánchez-Gómez, E. Effectiveness and safety of mepolizumab in severe refractory eosinophilic asthma; results in clinical practice. Drugs Context 2019, 8, 212584. [CrossRef] [PubMed]

26. Chung, K.F.; Adcock, I.M. Multifaceted mechanisms in COPD: Inflammation, immunity, and tissue repair and destruction. Eur. Respir. J. 2008, 31, 1334-1356. [CrossRef] [PubMed]

27. Kurakula, K.; Hamers, A.A.; van Loenen, P.; de Vries, C.J. 6-Mercaptopurine reduces cytokine and MUC5AC expression involving inhibition of NF-KB activation in airway epithelial cells. Respir. Res. 2015, 16, 73. [CrossRef]

28. Ghosh, S.; Erzurum, S.C. Nitric oxide metabolism in asthma pathophysiology. Biochim. Biophys. Acta 2011, 1810, 1008-1016. [CrossRef]

29. Lowry, J.L.; Brovkovych, V.; Zhang, Y.; Skidgel, R.A. Endothelial nitric-oxide synthase activation generates an inducible nitric-oxide synthase-like output of nitric oxide in inflamed enthothelium. J. Biol. Chem. 2013, 288, 4174-4193. [CrossRef]

30. de Andres, M.C.; Takahashi, A.; Oreffo, R.O. Demethylation of an NF-kB enhancer element orchestrates iNOS induction in osteoarthritis and is associated with altered chondrocyte cell cycle. Osteoarthr. Cartil. 2016, 24, 1951-1960. [CrossRef]

31. Blaise, G.A.; Gauvin, D.; Gangal, M.; Authier, S. Nitric oxide, cell signaling and cell death. Toxicology 2005, 208, 177-192. [CrossRef] [PubMed]

32. Lim, J.C.; Goh, F.Y.; Sagineedu, S.R.; Yong, A.C.; Sidik, S.M.; Lajis, N.H.; Wong, W.S.; Stanslas, J. A semisynthetic diterpenoid lactone inhibits NF-kB signaling to ameliorate inflammation and airway hyperresponsiveness in a mouse asthma model. Toxicol. Appl. Pharmacol. 2016, 302, 10-22. [CrossRef] [PubMed] 
33. Shin, I.S.; Hong, J.M.; Jeon, C.M.; Shin, N.R.; Kwon, O.K.; Kim, H.S.; Kim, J.C.; Oh, S.R.; Ahn, K.S. Diallyl-disulfide, an organosulfur compound of garlic, attenuates airway inflammation via activation of the Nrf-2/HO-1 pathway and NF-kappaB suprresion. Food Chem. Toxicol. 2013, 62, 506-513. [CrossRef] [PubMed]

34. Baird, L.; Dinkova-Kostova, A.T. The cytoprotective role of the Keap1-Nrf2 pathway. Arch. Toxicol. 2011, 85, 241-272. [CrossRef]

35. Cheng, H.T.; Yen, C.J.; Chang, C.C.; Huang, K.T.; Chen, K.H.; Zhang, R.Y.; Lee, P.Y.; Miaw, S.C.; Huang, J.W.; Chiang, C.K.; et al. Ferritin heavy chain mediates the protective effect of heme oxygenase-1 against oxidative stress. Biochim. Biophys. Acta 2015, 1850, 2506-2517. [CrossRef]

36. Han, C.W.; Kwun, M.J.; Kim, K.H.; Choi, J.Y.; Oh, S.R.; Ahn, K.S.; Lee, J.H.; Joo, M. Ethanol extract of Alismatis Rhizoma reduces acute lung inflammation by suppressing NF-kB and activating Nrf2. J. Ehtnopharmacol. 2013, 146, 402-410. [CrossRef]

37. Nikkhah, E.; Heshmati Afshar, F.; Babaei, H.; Asgharian, P.; Delazar, A. Phytochemical analysis and in-vitro bioactivity of Scrophularia umbrosa Rhizome (Scrophulariaceae). Iran. J. Pharm. Res. 2018, 17, 685-694.

38. Jeong, J.; Wahyudi, L.D.; Keum, Y.S.; Yang, H.; Kim, J.H. E-p-methoxycinnamoyl- $\alpha$-l-rhamnopyranosyl ester, a phenylpropanoid isolated from Scrophularia buergeriana, increases nuclear factor erythroid-derived 2-related factor 2 stability by inhibiting ubiquitination in human keratinocytes. Molecules 2018, $23,768$. [CrossRef]

39. Ding, B.; Lu, Y.; Li, Y.; Zhou, W.; Qin, F. Efficacy of treatment with montelukast, fluticasone propionate and budesonide liquid suspension for the prevention of recurrent asthma paroxysms in children with wheezing disorders. Exp. Ther. Med. 2019, 18, 3090-3094. [CrossRef]

(C) 2020 by the authors. Licensee MDPI, Basel, Switzerland. This article is an open access article distributed under the terms and conditions of the Creative Commons Attribution (CC BY) license (http://creativecommons.org/licenses/by/4.0/). 